\title{
Avaliação do conhecimento de acadêmicos de Farmácia sobre plantas medicinais e fitoterápicos
}

Assessment of knowledge of Pharmacy students about medicinal plants and herbal medicines Evaluación de conocimientos de los estudiantes de Farmacia sobre plantas medicinales

\author{
y medicamentos a base de hierbas \\ Lidia Kazue IUKAVA ${ }^{1}$ \\ Carlos Daniel de Siqueira CORADETTE ${ }^{2}$ \\ Priscila Megda Joao Job $\mathbf{Z A G O}^{3}$ \\ Fabiane Lucila MEOTTI ${ }^{4}$ \\ Heris Lorenzi dos Santos PERFEITO 5 \\ Joice Karina OTENIO ${ }^{6}$ \\ Ezilda JACOMASSI ${ }^{7}$
}

Daniela de Cassia Faglioni BOLETA-CERANTO7

${ }^{1}$ Biomédica, Mestrado Profissional em Plantas Medicinais e Fitoterápico na atenção Básica Unipar Universidade Paranaense.

${ }^{2}$ Cirurgião dentista especialista em saúde pública, e ortodontia

${ }^{3}$ Médica cardiologista, Mestrado Profissional em Plantas Medicinais e Fitoterápicos na atenção básica, Unipar

${ }^{4}$ Farmacêutica / Universidade Paranaense-Unipar/Especialista em Farmacologia pela Uninter/ Departamento de Saúde

${ }^{5}$ Fisioterapeuta Mestrado Profissional em Plantas Medicinais e Fitoterápico na atenção Básica Unipar Universidade Paranaense

${ }^{6}$ Mestre em Plantas Medicinais e Fitoterápicos na Atenção Básica. Universidade Paranaense, Umuarama - PR, Brasil

${ }^{7}$ Professoras no Programa de Mestrado Profissional em Plantas Medicinais e Fitoterápicos na Atenção Básica,

Universidade Paranaense - Umuarama - PR, Brasil

\section{Resumo}

O objetivo deste estudo foi avaliar o conhecimento sobre plantas medicinais e medicamentos fitoterápicos em uma instituição de ensino superior da Região Sul do Brasil a Universidade Paranaense - UNIPAR, considerando a importância da fitoterapia para a formação profissional dos cursos de Farmácia. Foi desenvolvido, um estudo quantitativo e descritivo, aplicando um questionário, pelo Google Formulários, para avaliar o conhecimento sobre as plantas e fitoterápicos, com todos os 23 acadêmicos do $4^{\circ}$ ano do curso de farmácia. Os resultados demonstraram que $60,9 \%$ dos alunos faz uso de alguma planta medicinal, 95,7\% dos acadêmicos aprendeu utilizar plantas medicinais na faculdade e as plantas mais citadas foram: hortelã, hibisco e boldo, 95,7\% respondeu que indicaria uso de fitoterápicos e plantas medicinais para outras pessoas, $87 \%$ dos acadêmicos responderam que talvez faça uma especialização em plantas medicinais e fitoterápicos. Este estudo demonstra que os acadêmicos, utilizam plantas medicinais com certa frequência, adquiriram informações provenientes da Universidade e do saber tradicional adquirido, havendo a necessidade de maior conhecimento sobre a importância do aperfeiçoamento profissional, com a realização de especialização, considerando o potencial da fitoterapia.

Descritores: Plantas Medicinais; Medicamentos Fitoterápicos; Educação em Farmácia.

\section{Abstract}

The aim of this study was to evaluate the knowledge about medicinal plants and herbal medicines in a higher education institution in the southern region of Brazil, Universidade Paranaense - UNIPAR, considering the importance of herbal medicine for the professional training of pharmacy courses. A quantitative and descriptive study was developed, applying a questionnaire, by Google Forms, to assess the knowledge about plants and herbal medicines, with all 23 academics from the 4th year of the pharmacy course. The results showed that $60.9 \%$ of students use some medicinal plant, $95.7 \%$ of students learned to use medicinal plants in college and the most cited plants were: mint, hibiscus and boldo, $95.7 \%$ answered that they would indicate use from herbal medicines and medicinal plants to other people, $87 \%$ of academics responded that perhaps they do a specialization in medicinal plants and herbal medicines. This study demonstrates that academics, using medicinal plants with a certain frequency, acquired information from the University and acquired traditional knowledge, with the need for greater knowledge about the importance of professional improvement, with the realization of specialization, considering the potential of herbal medicine.

Descriptors: Plants, Medicinal; Phytotherapeutic Drugs; Education, Pharmacy.

\section{Resumen}

El objetivo de este estudio fue evaluar el conocimiento sobre plantas medicinales y medicinas a base de hierbas en una institución de educación superior en la región sur de Brasil, la Universidade Paranaense - UNIPAR, considerando la importancia de la fitoterapia para la formación profesional de los cursos de farmacia. Se desarrolló un estudio cuantitativo y descriptivo, aplicando un cuestionario, de Google Forms, para evaluar los conocimientos sobre plantas y medicinas a base de hierbas, con los 23 académicos del $4^{\circ}$ año del curso de farmacia. Los resultados mostraron que el $60.9 \%$ de los estudiantes usa alguna planta medicinal, el $95.7 \%$ de los estudiantes aprendió a usar plantas medicinales en la universidad y las plantas más citadas fueron: menta, hibisco y boldo, el $95.7 \%$ respondió que indicaría uso de hierbas medicinales y medicinales. plantas a otras personas, el $87 \%$ de los académicos respondió que tal vez hacen una especialización en plantas medicinales y medicinas a base de hierbas. Este estudio demuestra que los académicos, utilizando plantas medicinales con cierta frecuencia, adquirieron información de la Universidad y adquirieron conocimientos tradicionales, con la necesidad de un mayor conocimiento sobre la importancia de la superación profesional, con la realización de la especialización, considerando el potencial de la fitoterapia.

Descriptores: Plantas Medicinales; Medicamentos Fitoterápicos; Educación en Farmacia.

INTRODUÇÃO

$$
\text { Desde as primeiras civilizações, as }
$$
plantas medicinais são reconhecidas como uma poderosa terapia para cura de doenças e o conhecimento proveniente delas se manteve preservado de geração em geração ${ }^{1,2}$. Por muito tempo, as plantas foram a única opção terapêutica para o tratamento das enfermidades existentes $^{3}$. Entretanto, com a evolução das tecnologias e da ciência, foram iniciadas novas abordagens em saúde, nas quais os medicamentos sintéticos e químicos se tornaram a primeira opção de tratamento e as plantas medicinais foram deixadas em segundo plano ${ }^{4}$. 
Com o intuito de retomar a utilização das plantas medicinais e incentivar a produção de medicamentos fitoterápicos no Brasil, em 2006 foi criada a Política Nacional de Plantas Medicinais e Fitoterápicos (PNPMF), que visa o desenvolvimento nas etapas de cultivo, secagem e processamento de plantas, distribuição de forma a fortalecer o sistema de saúde, garantir 0 acesso à matéria-prima vegetal de qualidade com eficácia e segurança, compilando com a valorização e preservação da biodiversidade brasileira, além de favorecer a indústria nacional ${ }^{5}$.

Dessa forma, se observa um aumento nas pesquisas científicas com relação ao estudo das espécies vegetais, que vão desde a identificação das estruturas químicas até a sua aplicabilidade na saúde ${ }^{6}$. Estes estudos, envolvem principalmente a etnobotânica, etnofarmacologia, fitoquímica, farmacologia e toxicologia, com ensaios pré-clínicos in vitro e in vivo com animais de experimentação e clínicos em humanos ${ }^{7}$.

Um problema encontrado com a utilização de plantas medicinais é a falta de conhecimento técnico-científico, pois, muitas pessoas desconhecem os riscos à saúde ${ }^{8}$. As plantas, possuem em sua composição compostos químicos, que podem se tornar tóxicos ao organismo humano, se utilizado de maneira incorreta. Nesse sentido, se faz necessário, para aplicação deste recurso terapêutico, a supervisão de um profissional de saúde capacitado para orientar o uso correto e os possíveis efeitos indesejáveis ${ }^{9,10}$.

Dentre os profissionais de saúde, os farmacêuticos são acessíveis à população e possuem conhecimento específico, para a prescrição de plantas medicinais e de medicamentos fitoterápicos, de acordo com 0 Art. 5 da Resolução n 586 de 29 de agosto de 2013, além disso, devem realizar a orientação aos usuários, incluindo a forma de utilização, posologia, tempo de tratamento, prevenir potenciais danos à saúde e devem monitorar a resposta terapêutica ${ }^{11,12}$.

Baseado nas políticas públicas que objetiva oferecer acesso às Plantas Medicinais e Fitoterápicos em todos os âmbitos da saúde, é importante que os profissionais da área estejam cientes sobre o assunto, principalmente, os farmacêuticos que também podem prescrever estes medicamentos. Sendo assim, o objetivo deste estudo foi avaliar o conhecimento sobre plantas medicinais e medicamentos fitoterápicos em uma instituição de ensino superior da Região Sul do Brasil a Universidade Paranaense UNIPAR, considerando a importância da fitoterapia para a formação profissional dos cursos de Farmácia.

MATERIAL E MÉTODO

O estudo se classifica como exploratório e descritivo. A pesquisa se volta para a investigação de valores e percepções, na qual se utilizou para coleta de dados aplicação de formulário do Google Forms. O formulário foi disponibilizado por meio de um link do Google Forms no grupo do Whatsapp dos acadêmicos, do $4^{\circ}$ ano do curso de graduação em Farmácia da Universidade Paranaense, unidade de Umuarama situada no estado do Paraná.

O formulário abrangeu perguntas, direcionadas ao conhecimento, forma de utilização, qual ou quais plantas são utilizadas, como adquiriu conhecimento sobre as plantas, qual o local de aquisição, se indicaria fitoterápicos e pretensão em realizar uma especialização em fitoterapia, além de informações sociodemográficas (sexo e idade). O estudo foi realizado respeitando os princípios éticos, sendo apreciado e aprovado pelo comitê de ética envolvendo seres humanos (Parecer: 4.110.675), em que se manteve o sigilo dos voluntários, sendo que estes possuíam total liberdade para recusar participar da pesquisa. Os dados obtidos, foram apresentados em frequências e percentuais distribuídos em gráficos e tabelas.

RESULTADOS E DISCUSSÃO

Foram voluntários todos os alunos formandos do curso de Farmácia da Universidade Paranaense, unidade de Umuarama - PR. A amostra foi composta por 23 alunos, sendo $43,5 \%$ (10) sexo masculino e $56,5 \%$ (13) feminino, com faixa etária entre 20 a 31 anos de idade.

A utilização das espécies vegetais, in natura ou produto tecnicamente elaborado, está presente no cotidiano de muitas pessoas, com o objetivo de restaurar a saúde. Entretanto, as espécies medicinais devem ser utilizadas com cautela e conhecimento específico. A Figura 1 demonstra o percentual de respostas, sobre o grau de conhecimento que os acadêmicos possuíam sobre as plantas medicinais. Observa-se, que a maioria das respostas foram acima de 6 , exceto um dos acadêmicos que assinalou possuir grau 5 de conhecimento.

O Brasil possui um conhecimento popular, muito vasto em plantas medicinais, resultado de uma miscigenação cultural originário dos continentes africanos, europeus e indígenas, com introdução de espécies exóticas, trazidas pelos colonizadores e escravos ${ }^{13}$.

Em relação ao uso de plantas medicinais 
na amostra pesquisada, foi verificado que $60,9 \% \quad(n=14)$ fazem uso de alguma planta medicinal (Figura 2).

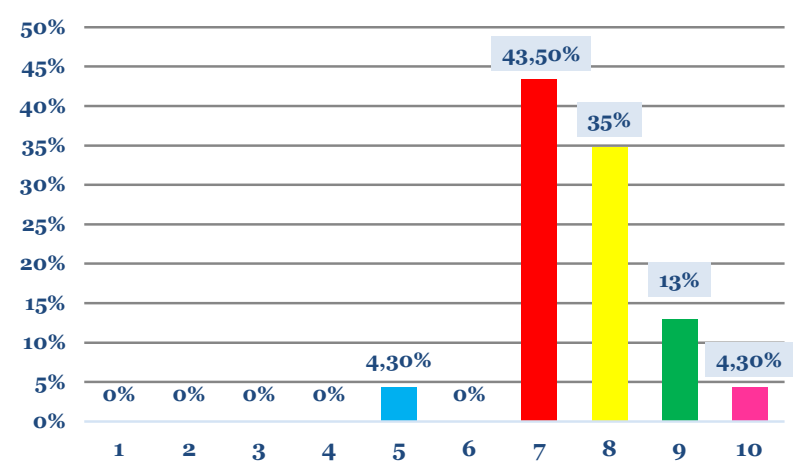

Figura 1: Grau de conhecimento sobre o uso de plantas medicinais de 0 a 10 (sendo zero nenhum e 10 total).

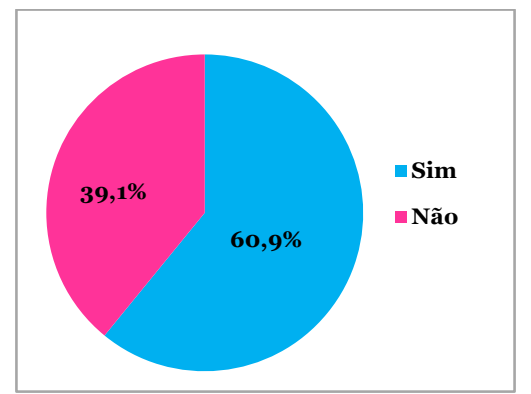

Figura 2: Uso de plantas medicinais pela amostra

Com relação à pergunta sobre qual planta utilizou e para qual enfermidade, apenas 14 pessoas responderam. Duas citações foram do produto Relora® um fitofármaco, constituído das cascas de Magnólia (Magnolia officinalis) e Phellodendron (Phellodendron amurense), plantas medicinais utilizadas para reduzir 0 estresse e a ansiedade ${ }^{14}$. Dois entrevistados responderam que utilizam 0 boldo para digestão. A camomila foi citada por 3 vezes, no tratamento de ansiedade e para cólicas. Um entrevistado respondeu que utilizou o hibisco, erva-java, dente-de-leão, mulungu e garcinia como diurético. Foi citado apenas uma vez, a cidreira para dor de estômago, a castanha-daíndia para circulação e melissa para insônia e ansiedade. O hibisco foi citado duas vezes, a hortelã uma vez, porém ambos sem relato de qual situação foi usado.

O hibisco, Hibiscus sabdariffa, é uma planta muito divulgada para perder peso, por evitar o acúmulo de gordura. Pesquisas científicas, realizadas com o chá de hibisco, afirmam sua ação na redução da adipogênese, contém em suas propriedades, bioativos que auxiliam na diminuição dos níveis de lipídios totais, colesterol e triglicerídeos, além disso, auxilia em distúrbios gastrointestinais, em cálculo renais, protege danos ao fígado e melhora efeitos da embriaguez ${ }^{15}$. Em estudo de Uyeda, relata que mulheres entre os 30 anos, são as que mais procuram e utilizam o chá de hibisco com o intuito de emagrecer ${ }^{16}$.

Foi questionado, se os entrevistados utilizaram plantas medicinais no período de 15 a 90 dias, anteriores à data da pesquisa, a esta questão $52,2 \%$ (12) responderam sim e 47,8\% (11) responderam não. Entre as citações, de quais espécies utilizaram, estava o "hibisco, erva-java, dente-de-leão, mulungu e garcinia para emagrecimento", "boldo para digestão", "cidreira para dor de estômago", "camomila contra cólicas", "castanha-da-índia para problemas circulatórios", "camomila em transtornos de ansiedade", "melissa para insônia e ansiedade", "guaco para tosse e expectoração" e "babosa como hidratante capilar". Além disso, um medicamento fitoterápico foi citado, o Kaloba® proveniente das raízes de Pelargonium sidoides D.C. utilizado contra infecções respiratórias.

Para obter informações referentes ao conhecimento adquirido sobre as plantas medicinais, foi questionado como aprenderam a utilizá-las. Nesta questão, os alunos poderiam assinalar mais de uma resposta. As opções foram, conhecimento na faculdade, com os pais, com os avós, com os vizinhos e outros (Figura 3).

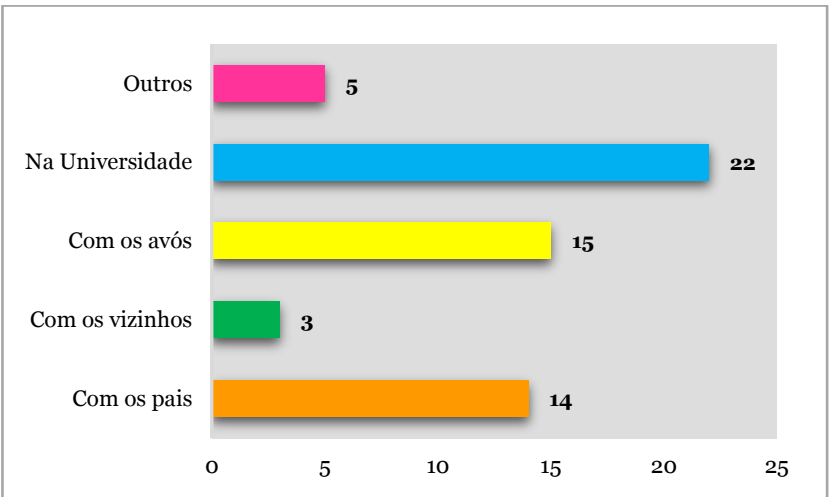

Figura 3: Distribuição percentual das respostas dos acadêmicos sobre o conhecimento adquirido das plantas medicinais.

Como resultado, responderam que aprenderam a utilizar as plantas medicinais na Universidade $95,7 \%$ (22), com os pais $65,2 \%$ (14), com avós $60,9 \%$ (15), com os vizinhos $13 \%$ (3) e outros $21,7 \%$ (5). O conhecimento proveniente da graduação, se explica pelo ensino da botânica e farmacognosia presentes na grade curricular do curso de farmácia das Faculdades e Universidades brasileiras ${ }^{17}$.

A Universidade Paranaense - UNIPAR, instituição na qual foi aplicado o questionário, possui projetos de extensão, que promove a utilização de plantas medicinais, orientações do uso correto das plantas, através de palestras em escolas, atua no processamento de 
produtos para a comunidade e na implementação de hortos medicinais em Unidades de Saúde. Esses trabalhos são realizados, desde 1996, quando foi implantado, em uma área de $30.000 \mathrm{~m}^{2}$, o projeto do Horto Medicinal da Universidade Paranaense, localizado em Umuarama - PR, contempla uma variedade significativa de espécies vegetais, medicinais, ornamentais, frutíferas, tóxicas, forrageiras e condimentares ${ }^{18}$. Além disso, o Horto Medicinal, fornece material vegetal, para aulas de graduação e pós-graduação, para 0 desenvolvimento de pesquisas científicas e atende a comunidade local e regional ${ }^{18}$. Essas atividades são fundamentais para 0 enriquecimento do conhecimento a respeito das plantas pelos futuros farmacêuticos, entrevistados neste trabalho.

Outra forma de adquirir conhecimento, muito citada pelos entrevistados, foi por meio do conhecimento tradicional adquirido sobre as plantas medicinais, ou seja, aquele transmitido de geração em geração, de extrema importância para a preservação das informações acerca das espécies vegetais ${ }^{1}$. Esse tipo de conhecimento, prevalece até os dias atuais, e tem despertado o interesse de pesquisadores, para a realização de estudos etnobotânicos e etnofarmacológicos, que buscam o resgate de saberes tradicionais, que são fundamentais para o desenvolvimento de novos produtos de origem vegetal ${ }^{2}$.

Foi investigado qual o local de aquisição das plantas: quintal de casa, em lojas de produtos naturais, com amigos, vizinhos ou outros (Figura 4). Os participantes também puderam assinalar mais de uma opção.

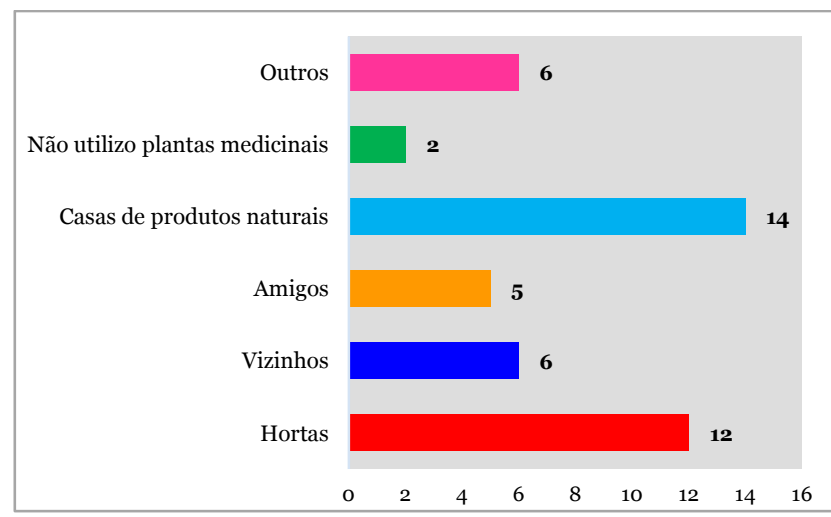

Figura 4: Aquisição das espécies vegetais.

O mercado de produtos naturais está em constante crescimento mundial, especialmente pela busca por práticas de vida mais saudáveis. Somado a isso, têm se buscado utilizar produtos livres de constituintes químicos, que atendam às necessidades nutricionais e alimentares, de maneira que os produtos naturais obtidos de processamento sustentável proporcionam a completa integração da saúde ${ }^{19}$. Essas são medidas que contribuem significativamente para a expansão da utilização de plantas medicinais e fitoterápicos.

Dessa forma, a grande procura por produtos naturais, leva as indústrias farmacêuticas, a investir em inovação e tecnologias, para a produção de medicamentos fitoterápicos ou de produtos tradicionais fitoterápicos. Com o objetivo de disponibilizar à sociedade, 0 acesso desse recurso terapêutico com mais eficácia, segurança e qualidade ${ }^{20}$.

Foi questionado que plantas medicinais os acadêmicos costumam utilizar, conforme mostra a Figura 5.

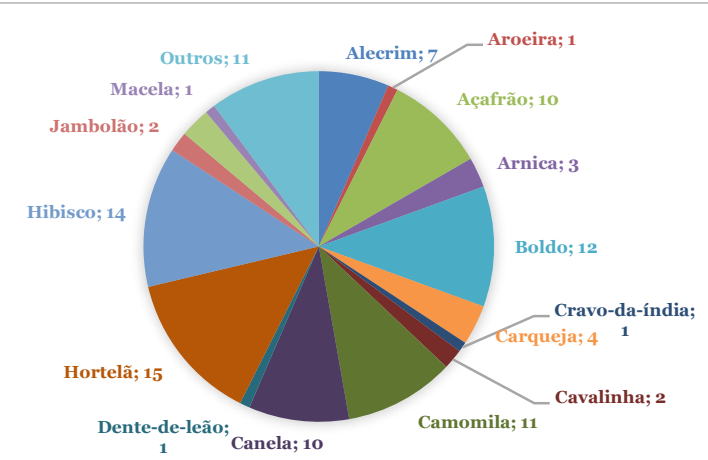

Figura 5: Plantas medicinais utilizadas pelos acadêmicos do $4^{\circ}$ ano do curso de Farmácia (os numerais ao lado da planta corresponde ao número de alunos que as citaram)

Em pesquisa etnobotânica realizada por Oliveira e Menini ${ }^{21}$ a planta com o maior número de citações de utilização pelos entrevistados foi hortelã, e a indicação terapêutica foi como vermífugo, digestório e calmante.

O boldo é uma planta muito citada em diversos textos da farmacopeia pelo seu uso contra dispepsias, náuseas e constipação intestinal $^{22}$. Suas folhas são usadas na medicina tradicional para tratamento de problemas digestivos, hepáticos e colelitíase, tendo ainda propriedades diuréticas e anti-inflamatórias ${ }^{23}$. Apesar de possuir várias finalidades terapêuticas, um estudo realizado com extratos das folhas mostrou ação abortiva e teratogênica em ratos ${ }^{24}$. As espécies mais comumente utilizadas no Brasil são Peumus boldus Molina (boldo-do-chile), da família Monimiaceae; Coleus barbatus (Andrews) Benth., (falso-boldo ou boldo-brasileiro), da família Lamiaceae; Acmella ciliata (Kunth) Cass. (boldo-baiano), da família Asteraceae ${ }^{25}$.

A Curcuma longa L., é uma planta muito usada na culinária, apreciada por seu valor nutricional, ganhou espaço por suas propriedades medicinais que incluem: atividade hepática, gastroprotetora, anti-inflamatória, antimicrobiana, anti-HIV, hipoglicemiante, antiagregante, dermatológica, oftalmológica, 
antioxidante, imunomoduladora, no sistema respiratório, no sistema reprodutor, no sistema digestório e no sistema nervoso central e uma planta potencial que está sendo muito estudada e divulgada pelo seu potencial farmacológico ${ }^{26}$.

A canela, Cinnamomum zeylanicum, é uma planta aromática, muito conhecida pelo grande potencial fungicida e bactericida, além disso, é anti-séptica, adstringente, afrodisíaca, carminativa, digestiva, estimulante, sedativa e tônica ${ }^{27}$. Contudo, esta espécie vegetal, deve ser utilizada com cautela, principalmente por possuir bioativos com atividade hipertensora, prejudicial às pessoas propensas à hipertensão arterial $^{27}$.

A camomila, Matricaria recutita L., é muito utilizada na medicina tradicional, no tratamento de ansiedade, na forma de chá por infusão das flores secas e também na forma de medicamento fitoterápico. Tendo como principal mecanismo de ação, da atividade ansiolítica, a inibição de receptores acetil-coenzima- ${ }^{28}$.

Foi questionado se os futuros profissionais indicariam o uso de fitoterápicos e plantas medicinais (Figura 6). Responderam que indicaria o uso de fitoterápicos e plantas medicinais para outras pessoas 95,7\% e responderam talvez 4,3\%. Em estudo, realizado por Faria et al. ${ }^{20} \mathrm{com}$ acadêmicos do núcleo de ciências biológicas, incluindo os alunos de farmácia, obtiveram nos resultados, $80,3 \%$ de confirmação para indicação de plantas medicinais e fitoterápicos como forma de tratamento.

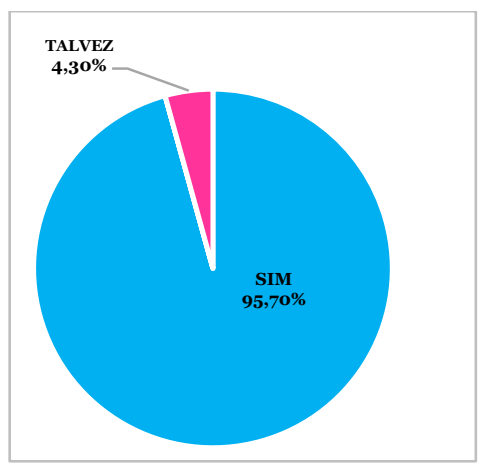

Figura 6: Indicação do uso de fitoterápicos e planta medicinais.

De acordo com os acadêmicos, estes indicariam plantas medicinais e fitoterápicos por apresentar efeito terapêutico, menos eventos adversos e por ser uma opção de tratamento mais acessível à população. "Sim, pois acho um ótimo benefício tanto em preço quanto para a saúde", "Porque podem fazer o mesmo efeito de medicamentos sintéticos e com o menor custo", "Indico pelo efeito benéfico e menor contraindicação", "Sim, pois eu tive resultado positivo no uso", "Pois é excelente custo benefício e com efeitos menores comparados com tratamento convencionais".

A Figura 7 mostra a distribuição percentual da pretensão dos acadêmicos em realizar no futuro uma especialização em fitoterapia, já que os profissionais farmacêuticos podem prescrever plantas medicinais $\mathrm{e}$ fitoterápicos, $87 \%$ responderam talvez, 8,7\% responderam $\operatorname{sim}$ e $4,3 \%$ responderam não. Os resultados demonstram que a fitoterapia, uma área da profissão farmacêutica, ainda é pouco explorada. Além disso, a população não está habituada com as consultas e prescrições farmacêuticas.

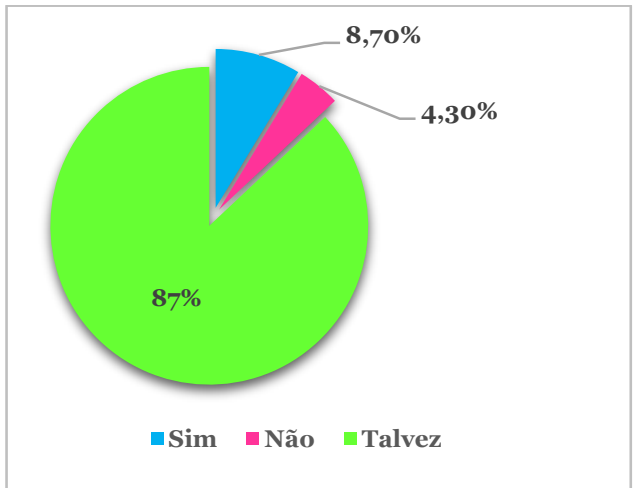

Figura 7: Distribuição percentual das respostas dos participantes da pesquisa sobre a pretensão em realizar especialização futura em plantas medicinais e fitoterápicos.

O farmacêutico está inserido ao Sistema Único de Saúde, podendo desempenhar papel fundamental para o melhor funcionamento do setor público, diminuindo as filas $e$ disponibilizando cuidados essenciais à saúde, visto que, estão em contato frequente com a população. Os farmacêuticos, capacitados poderão prescrever medicamentos fitoterápicos e plantas medicinais, além de, orientar sobre a utilização correta, possíveis atividades tóxicas e interações medicamentosas e alimentares ${ }^{17}$.

Como justificativa à resposta, descrita na Figura 7, os acadêmicos responderam: "Não sei ainda, pois ainda estou terminando minha graduação, não sei em que me especializar ainda", "Pode ser que futuramente me interesse", "Vou ser farmacêutico e vou trabalhar com esses produtos, confio neles e vou passar meu conhecimento adiante", "É uma área que vem crescendo muito, acho interessante".

A implantação da Política Nacional de Plantas medicinais, a partir do Decreto oㅜ 5.813, de 22 de junho de 2006, estabeleceu diretrizes para 0 fortalecimento da utilização e preservação da biodiversidade brasileira, além de incentivar com as pesquisas $e$ desenvolvimento de novos produtos vegetais. Para que toda a população possa realizar o acesso a este recurso natural, tão importante, com mais eficácia e segurança ${ }^{5}$. 
CONCLUSÃO

Nesta avaliação de conhecimento com os acadêmicos de farmácia conclui-se que $60,9 \%$ faz uso de alguma planta medicinal, $95,7 \%$ dos acadêmicos aprendeu utilizar plantas medicinais na Universidade. As plantas mais citadas foram: hortelã, hibisco utilizadas para perder peso e boldo para problemas gástricos. Além disso, 95,7\% respondeu que indicaria uso de fitoterápicos e plantas medicinais para outras pessoas, $87 \%$ dos acadêmicos responderam que talvez façam uma especialização em plantas medicinais e fitoterápicos. Considerando, a vasta biodiversidade brasileira e o potencial para a produção de medicamentos fitoterápicos, o aperfeiçoamento nesta área é muito promissor.

\section{REFERÊNCIAS}

1. Badke M, Heisler E, Ceolin S, Andrade A, Budó M, Heck R. O conhecimento de discentes de enfermagem sobre uso de plantas medicinais como terapia complementar. RPCFO. 2017;9(2):459-65.

2. Salesse D, Medeiros FC, Silva CCM, Lourenço ELB, Jacomassi E. Etnobotânica e etnofarmacologia das espécies de Amaryllidaceae, Anacardiaceae, Annonaceae e Apiaceae. Arq Ciênc Saúde UNIPAR. 2018;22(3):205-16.

3. Badke RM, Budó MLD, Silva FM, Ressel LB. Plantas medicinais: o saber sustentado na prática do cotidiano popular. Rev Esc Anna Nery. 2011;15(1):132-39.

4. Hasenclever L, Paranhos J, Costa CR, Cunha G, Vieira D. A indústria de fitoterápicos brasileira: desafios e oportunidades. Ciênc saúde colet. 2017;22(8):2559-69.

5. Brasil. Ministério da Saúde. Secretaria de Ciência, Tecnologia e Insumos Estratégicos e Departamento de Assistência Farmacêutica e Insumos Estratégicos. Portaria no 5.813, de 22 de junho de 2006. Aprova a Política Nacional de Plantas Medicinais e Fitoterápicos e dá outras providências. Diário Oficial da República Federativa do Brasil. Brasília. 2006;20, p. 60.

6. Firmo WCA, Menezes VJM, Passos CEC, DIAS CN, Alves LPL, Dias ICL. Contexto histórico, uso popular e concepção científica sobre plantas medicinais. Cad Pesq.2011;18:90-5.

7. Otenio JK, Baish RG, Carneiro VPP, Lourenço ELB, Alberton $O$, Soares $A A$ et al. Ethnopharmacology of Cuphea carthagenensis (Jacq.) JF Macbr: A review. Braz J of Develop 2020;6(3):10206-219.

8. Kovalski LM, Obara TA. O estudo da etnobotânica das plantas medicinais na escola. Rev Ciênc Educ. 2013;19(4):911-27.

9. Carneiro VPP, Gumy MP, Otenio JK, Bortoloti DS, Castro TE, Lourenço ELB et al. Perfil dos agentes comunitários de saúde de um município do estado do Paraná e sua relação com plantas medicinais. Braz $\mathrm{J}$ of Develop. 2020;6(1):2902-918.

10. Martelli A, de Carvalho LAHB. Percepção dos moradores do distrito de Eleutério, município de Itapira-SP, acerca da utilização de plantas medicinais. Arch Health invest.2019;8(2):79-84.

11. Brasil. RDC no 586 de 29 de agosto de 2013. Regula a prescrição farmacêutica e dá outras providências. Diário Oficial da União, Brasília. 2013.

12. Brasil. RDC no 546 de 21 de julho de 2011. Dispõe sobre a indicação farmacêutica de plantas medicinais e fitoterápicos isentos de prescrição e o seu registro. Diário Oficial da União, Brasília. 2011.

13. Melo JG, Martins JDGR, Amorim ELC, Albuquerque UP. Qualidade de produtos a base de plantas medicinais comercializados no Brasil: castanha-da-índia (Aesculus hippocastanum (L.), capim-limão (Cymbopogon citratus (DC.) Stapf e centela (Centella asiatica (L.) Urban). Acta Bot Bras. 2007;21(1):27-3.

14. Talbott SM, Talbott JA, Pugh M. Effect of Magnolia officinalis and Phellodendron amurense (Relora $\left.{ }^{\circledR}\right)$ on cortisol and psychological mood state in moderately stressed subjects. J Int Soc Sports Nutr. 2013;10(1):37.

15. Cunha MJ, Viana ESM, Souza JT, Silva SS. Os efeitos do Hibisco (Hibiscos sabdariffa) no emagrecimento. Rev Cient Univiçosa. 2016;8(1):657-61.

16. Uyeda M. Hibisco e o processo de emagrecimento: uma revisão da literatura Saúde em Foco. 2015;7:82-90.

17. Marques PA, Simão TA, Moriya MM, Antunes VMS, Oliveira CR. Prescrição farmacêutica de medicamentos fitoterápicos. BJNS. 2019;2(1): 1-9.

18. Canzi KN, Byczkovski C, Grigol DEB, Canezin M, Lima LT, Corrêa EJT et al. Levantamento florístico do horto medicinal do campus 2 da Universidade Paranaense (UNIPAR)Umuarama/PR. Arq ciências saúde Unipar. 2012;16(3):123-37.

19. Willerding AL, Silva LR, Silva RP, Assis GMO, Paula EVCM. Estratégias para o desenvolvimento da bioeconomia no estado do Amazonas. Estudos Avançados. 2020;34(98): 145-65.

20. Faria BMA, Valiati TB, Oliveira AA, Salvi JO. A Fitoterapia entre Acadêmicos Das Ciências da Vida. Saúde e Desenvolvimento. 2017;11(9): 200-13.

21. Oliveira ER, Menini Neto L. Levantamento etnobotânico de plantas medicinais utilizadas pelos moradores do povoado de Manejo, Lima Duarte-MG. Rev bras plantas med. 2012;14(2): 311-20. 
22. Marques CSA et al. Anatomia e análise de óleo essencial das folhas de Hennecartia omphalandra J. Poisson (Monimiaceae). Rev. bras farmacogn. 2009;19(1):95-105.

23. Ruiz ALTG, Taffarello D, Souza VHS, Carvalho JE. Farmacologia e toxicologia de Peumus Boldus e Baccharis genistelloide. Rev bras farmacogn. 2008;18(2):295-300.

24. Bakke A, Leite RS, Marques MFL, Batista LM. Estudo comparativo sobre o conhecimento do uso de plantas abortivas entre alunas da área de saúde e da área de humanas da Universidade Federal da Paraíba. REF. 2008;5(1):24-31.

25. Mauro C, Silva CP, Missima J, Ohnuki T, Rinaldi RB, Frota M. Estudo anatômico comparado de órgãos vegetativos de boldo miúdo, Plectranthus ornatus Codd. e malvariço, Plectranthus amboinicus (Lour.) Spreng. Lamiaceae. Rev bras farmacogn. 2008;18(4):608-13.

26. Marchi JP, Tedesco L, Melo AC, Frasson AC, Sato SW. Curcuma longa L., o açafrão da terra, e seus benefícios medicinais. Arq ciênc Saúde UNIPAR. 2016;20(3):189-94.

27. Gomes EMC, Firmino AV, Pena RCM, Almeida SSMS. Efeito inibitório in vitro de extratos de Cinnamomum zeylanicum Blume no controle de Cylindrocladium candelabrum. Ciênc Florest. 2018;28(4):1559-567.

28. Silva GAO. Conhecimento farmacológico e etnobotânico de alunos do primeiro ano do ensino médio, numa escola pública do Município de João Pessoa, Paraíba. Rev Bras Gest Amb Sustent. 2017;4(8):381-93.

\section{CONFLITO DE INTERESSES}

Os autores declaram não haver conflitos de interesse

\section{AUTOR PARA CORRESPONDÊNCIA}

Daniela de Cassia Faglioni Boleta-Ceranto

Praça Mascarenhas de Moraes, 4282 - Centro

87502-210 Umuarama - PR, Brasil

E-mail: dcboleta@prof.unipar.br

Submetido em 27/10/2020

Aceito em 16/07/2021 